\title{
Dynamics of composite nonlinear systems and materials for engineering applications and energy harvesting - The role of nonlinear dynamics and complexity in new developments
}

\author{
G. Litak ${ }^{1, a}$ and E. Manoach ${ }^{2, b}$ \\ 1 Faculty of Mechanical Engineering, Lublin University of Technology, Nadbystrzycka 36, \\ 20-618 Lublin, Poland \\ 2 Institute of Mechanics, BAS, Sofia 1113, Bulgaria
}

Received 4 June 2013 / Received in final form 2 August 2013

Published online 30 September 2013

\begin{abstract}
This engineering research focus issue was inspired by the workshop organised by us on March 19th 2012 at the Lublin University of Technology (in the framework of CEMCAST) titled "Dynamics of functionally graded materials and systems with hysteresis" The collection of the present research papers followed our fruitful discussions focussing on "Dynamics of composite nonlinear systems and materials for engineering applications and energy harvesting" which is now the title of the present volume. In the next two sections we briefly discuss the topics included in this focus issue.
\end{abstract}

\section{Nonlinear dynamics of engineering systems}

Structures made of advanced composite materials are widely used in many modern branches of industry due to advantageous balance between their weight, stiffness and strength. Such structures are very often subjected to operation and variable environmental conditions that lead to high nonlinear behaviour of the structures such as large deflections and rotations, contact phenomena, nonlinear damping, coupled fields (thermo mechanical; electro-mechanical), etc. On the other hand the nonlinear behaviour of such structures is often desired and designed by the engineers because it could allow smart and very effective behaviour of the designed structure. Typical examples in these directions are the shape memory alloys, magnetosctrictive, thermoelectric, piezoelectric devices used in system control and health monitoring, energy harvesting, smart sensors and actuators, self-sensing actuators used in medicine or industry and MEMS. Nonlinear effects are usually introduced into the above devices as they are necessary to increase their efficiency and/or improve their controllability. The focus of this issue of the journal aims to report on latest research and development findings related to the multi-disciplinary area of nonlinear dynamic phenomena

\footnotetext{
a e-mail: g.litak@pollub.pl

b e-mail: e.manoach@imbm.bas.bg
} 
in smart materials and related structures made from composite materials, functionally graded materials, laminated structures, and energy harvesting systems.

Friswell and Flores studied nonlinear phenomena in dynamic behaviour of beams with tunable geometric nonlinear stiffness. The objective was to produce a vibroisolator which is effective and much simpler than the existing isolators. Tuning is achieved by actuators at the ends of the beams that prescribe the axial displacement and rotation. Additionally, the authors have studied composite beams with an initial thermal pre-stress, resulting in internal stresses that give the required nonlinear response. Interesting phenomena in structures made by shape memory alloys (SMA) are studied in several papers. In the work of Bessa et al. an adaptive fuzzy sliding mode controller is proposed for vibration suppression in SMA two-bar truss. The model allows an appropriate qualitative description of system dynamics, which can exhibit chaotic behaviour. The main goal is the vibration reduction, avoiding some critical responses as snap-through behaviour. The adoption of a robust and adaptive control law allows simple constitutive models, such as the polynomial equation to be used for control purposes. The control system performance is demonstrated by numerical simulations. Anderson et al. used SMA to develop a biomedical deviceorgan positioner. The design includes multiple transverse loading experiments at different temperatures. The purpose of the simulations and experimental results was to develop a design envelope for the organ positioning device. A finite element model was used to extend the breadth of the positioner's working regime by simulating geometric perturbations and observing the response. It was found that overall geometry plays little role in the stiffness, but rather the diameter of the SMA element used in the flexion mode is the commanding feature. The dynamical response of the shape memory device (SMD) was considered by Litak et al. Instead of the standard Lyapunov exponent treatment, the statistical 0-1 test based on the asymptotic properties of a Brownian motion chain was successively applied to reveal the chaotic nature of trajectories in the special case in which temperature variations were neglected. In this work, the 0-1 test was applied to fully non-isothermal trajectories governed by hysteretic model of SMA. Complex dynamics of hysteretic systems were also studied by Radons and Zienet. They studied the magnetic pendulum as a dynamical system with hysteretic nonlinearity by the differential-operator equation with hysteretic damping. They found a fractal dependence of the asymptotic behaviour as a function of the initial conditions. The authors also applied the $0-1$ test to identify chaotic response. Lamarque et al. studied multi-scale energy exchanges between a nonlinear oscillator of Bouc-Wen type and another coupled nonlinear system. Their model was directly linked the hysteretic behaviour of materials or structures and the so called nonlinear energy sink. A strip with a crack made of functionally graded material subjected to a thermal shock is studied by Ivanov et al. Continuous functions were constructed for the Young's modulus, the thermal expansion coefficient, the thermal conductivity and the thermal diffusivity for functionally graded composite consisted of $\mathrm{Al}_{2} \mathrm{O}_{3}$ and $\mathrm{Al}$. The position-dependent properties of the material were obtained experimentally and implemented as user-defined material properties in

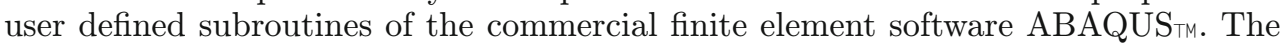
influence of the temperature dependence of the fracture toughness of the material and of the residual stress on the crack resistance were also studied. Results on the nonlinear oscillations of axially functionally graded beams were presented in the work of Babilio. The beam is axially loaded by a force containing a static part load, plus a timedependent harmonic part. The nonlinear phenomena like period doubling, chaos, and periodic windows in between two chaotic regions are studied by constructing bifurcation diagrams. The influence of gradation of the material on the behaviour of the beam is demonstrated. Nonlinear dynamic behaviour of a layered composite beam with delamination is studied experimentally and theoretically by Manoach et al. The delamination model considers the normal and shear forces arising 
during the contact interaction between sublaminates and the additional damping due to sublaminates friction. Special samples of multilayered beams have been created. Small parts of adjacent layers have been cut and replaced by inclusions from different materials thus modelling delamination. The experimental study confirmed the applicability of the model. Wang and Luo investigated periodic motions of a nonlinear rotating blade subjected to a time-varying torque. A gyroscopic system with parametric excitations was obtained for such a rotating blade. The study provides an efficient way to study the global dynamics of gyroscope systems with time-varying excitations.

\section{Nonlinear dynamics in energy harvesting}

Recently, the demands on portable devices, self-powered wireless sensors and similar applications that currently require rechargeable batteries, have increased considerably. Vibration energy is one of the candidate technologies, in competition with other possibilities such as photo-voltaic or thermal gradient approaches. The effectiveness of devices based on harvesting vibration energy and their availability are growing continuously. Ambient mechanical vibrations can be converted to the electrical energy using electromagnetic, piezoelectric, and electrostatic methods. Especially piezoelectric transducers can be easily integrated into elastic multilayer cantilever beams. The main advantage of the piezoelectric devices, compared to the electrostatic and electromagnetic alternatives, is their high power output for a given device volume and excitation. Although they are not suitable for generating high power levels, they are very good for applications to small electronic devices and sensors. The classic approach is to tune the mechanical part of the harvester to resonant. This approach is fairly good for narrow bands and stable excitations but it performs poorly in offresonance conditions or with variable ambient excitation. Various nonlinear effects can overcome this limitation providing the so called broadband frequency effect. By including nonlinear effects to the mechanical resonator one can obtain inclination of the resonance curve, double-well dynamics or self-excitation effects.

The rest of the papers deal with nonlinear effects in energy harvesting. Abdelkefi et al. considered galloping excitation of the mechanical resonator coupled to the piezoelectric beams subjected to an incoming flow with a speed higher than the critical value. They studied the harvesting energy efficiencies using the nonlinear normal forms of the dynamic system near galloping. Betts et al. provided experimental results on piezoelectric layers attached to a bistable asymmetric laminate. They studied the transitions between mode shapes with excitation frequencies to understand the dynamic response of the structure and power generation characteristics. Kecik and Borowiec studied the autoparametric system composed of the pendulum and electromagnetic transducer. The pendulum was suspended on a nonlinear spring and excited harmonically. They investigated the voltage output combined with different dynamical responses of the pendulum. Kucab et al. examined the energy harvesting system consisting of two different concentrated masses (with magnets) attached to piezoelastic beams, and driven by harmonic kinematic excitations. The nonlinearity of the system is achieved by interaction of magnets. A simple portal frame structure presented was suggested for energy harvesting by Iliuk et al. Their system was a non-ideal system (NIS) due to interaction with the energy source, a DC motor with limited power supply and the system structure. The nonlinearities presented in the piezoelectric material are considered in the piezoelectric coupling mathematical model. Vinolo et al. proposed a new idea of harvesting energy based on impacts of a pendulum to piezoelectric disk elements. The horizontally suspended physical pendulum was chosen as a mechanical resonator. The system was suggested to harvest 
energy from the see motion. The stationary response of nonlinear magnetopiezoelectric energy harvester systems under stochastic excitation was examined by Martens et al. Depending on the magnet positions and orientations the vibrating piezo beam system was characterised by different potentials. By considering random excitation, the authors solved the corresponding Fokker-Planck equation. Vocca et al. compared vibrational energy harvesting in two models: a simple nonlinear cantilever and buckled beams (bridge configuration). In both models the voltage output was maximum in the bistable region. For considered conditions, the cantilever configuration appeared to be more efficient than the bridge one. Zhang et al. studied coupling of an electric field and mechanical excitation on Ericsson-based cycles. By applying high electrical field levels (high amplitude, high frequency), it is possible to engage a non-linearity through the piezoceramic. A special dynamic hysteresis model was developed to provide a real estimation of the harvested energy. 